\title{
Magnetic relaxation in finite two-dimensional nanoparticle ensembles
}

\author{
S. I. Denisov* and T. V. Lyutyy \\ Department of Mechanics and Mathematics, Sumy State University, \\ 2, Rimskiy-Korsakov Street, 40007 Sumy, Ukraine \\ K. N. Trohidout \\ Institute of Materials Science, NCSR "Demokritos," 15310 Athens, Greece
}

\begin{abstract}
We study the slow phase of thermally activated magnetic relaxation in finite two-dimensional ensembles of dipolar interacting ferromagnetic nanoparticles whose easy axes of magnetization are perpendicular to the distribution plane. We develop a new method to numerically simulate the magnetic relaxation for the case that the smallest heights of the potential barriers between the equilibrium directions of the nanoparticle magnetic moments are much larger than the thermal energy. Within this framework, we analyze in detail the role that the correlations of the nanoparticle magnetic moments and the finite size of the nanoparticle ensemble play in magnetic relaxation.
\end{abstract}

PACS numbers: 75.50.Tt, 75.40.Mg, 76.20.+q 


\section{INTRODUCTION}

The role of the dipolar interaction in systems of nanometer-sized ferromagnetic particles, or nanoparticle ensembles for short, has been intensively studied in recent years. Such ensembles have numerous technological applications, and it is important to understand their magnetic phenomena and processes. ${ }^{1.2}$ One of the most complicated problems, where dipolar interactions must be taken into account, is thermally activated magnetic relaxation. To derive the law of magnetic relaxation, i.e., of the dimensionless reduced magnetization, usually requires the derivation of the distribution function of the nanoparticle magnetic moments. In the simplest case, that of non-interacting nanoparticles with conserved total magnetic moments, the distribution function obeys the Fokker-Planck equation $\underline{3}^{-}$For nanoparticle ensembles with more or less realistic magnetic energy, however, its time-dependent solutions are not known, and exact results were found mainly for numerical characteristic of the relaxation process such as the largest relaxation time. 3.4.5.6.7.8 $^{3}$

Unfortunately, in the case of dipolar interacting nanoparticles no exact results for the magnetic relaxation exist. This fact makes it difficult to check the validity of different approximate methods and approaches that are extensively used in this area.9.10.11.12.13.14.15.16.17 The justification of approximations is a very important task because the use of non-rigorous,

although plausible, approximations can lead to opposite conclusions. ${ }^{12.13}$ One expects that a sufficiently rigorous analysis of the relaxation law can be performed for the simplest systems like two-dimensional (2D) ensembles of identical, spherical nanoparticles with conserved magnetic moments and large uniaxial perpendicular anisotropy. Such ensembles represent an important class of perpendicular magnetic recording media,,$\stackrel{18}{,}$ and they are convenient systems to study experimentally and theoretically the role that the dipolar interaction plays in magnetic relaxation.

Magnetic relaxation in such ensembles was considered first by Lottis, White, and Dahlberg $\frac{19}{}$ within the simplified version of the mean-field approximation. Using the concept of a demagnetizing field, the authors wrote down the equation that describes the relaxation of magnetization from the initial state, when all nanoparticle magnetic moments are oriented along a certain direction of the easy axis, to the demagnetized ground state. They solved this equation numerically and showed that for a limited time domain relaxation occurs slower than the Debye model predicts. They approximated the relaxation law $\rho(t)$ by 
a stretched-exponential dependence, which, however, does not hold for all times.

Recently, we studied the influence of the mean and the fluctuating components of the dipolar field on the process of magnetic relaxation in those ensembles. ${ }^{20,21}$ Using the FokkerPlanck equation, we derived an equation that describes the so-called slow relaxation, i.e., relaxation for times exceeding the time $t_{q e}$ to establish the quasiequilibrium distribution of the magnetic moments $\left(t_{q e} \sim 10^{-8} \mathrm{~s}\right.$, see Sec. II B), and we solved it in limiting cases. We showed that both the mean and the fluctuating components of the dipolar field enhance relaxation, and that for small and large times magnetic relaxation has a Debye character, but the corresponding relaxation times can be very different. This difference causes the quasi-logarithmic relaxation at intermediate times that was found numerically in Ref. 19.

The role that the correlations of directions of the nanoparticle magnetic moments play in magnetic relaxation has not yet been clarified. Clearly, correlation effects are very significant, and we expect that, due to the antiferromagnetic character of the dipolar interaction in such ensembles, they can qualitatively change the relaxation law. The influence of the finite size of the nanoparticle ensemble on magnetic relaxation is another important problem, which also has not yet been addressed. We expect that, due to the long-range character of the dipolar interaction, magnetic relaxation will significantly depend on the ensemble size, especially for small times when dipolar fields near the internal and the external magnetic moments can be quite different.

The complexity of these problems forces us to seek numerical solutions. The known methods of numerical simulation of magnetic relaxation, such as directly integrating the stochastic Landau-Lifshitz equation, ${ }_{22,23,24}$ the conventional Monte Carlo method, $\stackrel{25,26}{,}$ and the time quantified Monte Carlo method 27 are not suitable for our purposes. The main reasons are the following. To integrate the Landau-Lifshitz equation, the integration time step must be smaller than the inverse of the precession frequency of the nanoparticle magnetic moments $\left(\sim 10^{-11} \mathrm{~s}\right)$. Therefore, this method usually works only for the description of the fast magnetic relaxation, i.e., relaxation on time scales smaller than $t_{q e}$. The conventional Monte Carlo method is not suitable, since each Monte Carlo step has no physical time associated with it. The time quantified Monte Carlo method also cannot be applied to our situation; the number of Monte Carlo steps that are necessary to calculate the relaxation law on times comparable with the relaxation time becomes prohibitively large in the case of high potential barriers between the equilibrium directions of the magnetic moments (see 
Sec. III B). Further, that method is valid only in the high damping limit, i.e., if there is no precession of the magnetic moments.

In this paper we develop a new method to numerically simulate thermally activated magnetic relaxation in finite 2D ensembles of dipolar interacting ferromagnetic nanoparticles. We consider the case where the nanoparticles with uniaxial anisotropy occupy the sites of a square lattice and their easy axes of magnetization are perpendicular to the lattice plane. We develop an equation that relates the magnetization of the ensemble at the next time step to the known state of the nanoparticle ensemble at the previous time step, and a numerical procedure that defines the ensemble state at the next time step. To derive the

probability densities for the reorientation of the nanoparticle magnetic moments, contained in this equation, we exploit that they can be represented via the mean times for magnetic moments to reorient, or, in other words, via the so-called mean first-passage times, and calculate these times using the backward Fokker-Planck equation.

The paper is organized as follows. In Sec. II, we introduce the equation mentioned above and derive rigorous expressions for the probability densities of reorientation of the nanoparticle magnetic moments. The algorithm for the numerical calculation of the relaxation law is described in Sec. III. In the same section we present the numerical results and analyze the features of the magnetic relaxation caused by both the correlations of the nanoparticle magnetic moments and the finiteness of the nanoparticle ensemble. We summarize our results in Sec. IV.

\section{ANALYTICAL RESULTS}

We consider a system of $N$ uniaxial and identical spherical ferromagnetic nanoparticles with a radius $r$. We assume that the nanoparticle centers occupy the sites of a square lattice of size $L d \times L d\left[(L+1)^{2}=N\right]$ and lattice spacing $d(\geq 2 r)$. The easy axes of nanoparticles magnetization are perpendicular to the lattice plane ( $x y$-plane), and at the initial time $t=0$ all magnetic moments $\mathbf{m}_{i}(t)$ (the index $i$ labels the nanoparticles) are oriented along the $z$ axis (see Fig. 1). We also assume that the smallest heights $\Delta U_{i}$ of the potential barriers between the equilibrium directions of the nanoparticle magnetic moments are much larger than the thermal energy $k_{B} T$ ( $k_{B}$ is the Boltzmann constant, $T$ is the absolute temperature), i.e., the condition $\varepsilon_{i}=\Delta U_{i} / k_{B} T \gg 1$ holds for all nanoparticles. The main goal of this 
section is to find the relation between the reduced magnetization at times $t$ and $t+\tau$.

\section{A. Equation for the reduced magnetization}

For $\varepsilon_{i} \gg 1$, the vectors $\mathbf{m}_{i}(t)$ fluctuate within small vicinities of the positive and negative directions of the $z$-axis, and they are reoriented only rarely. Consequently, the average numbers of positively and negatively oriented magnetic moments have well-defined values $N_{+}(t)$ and $N_{-}(t)$, respectively, at any instant $t$. Since the number of magnetic moments that at time $t$ have reoriented is much less than $N$, the approximate relation $N_{+}(t)+N_{-}(t) \approx N$ holds, and we can define the reduced magnetization of the nanoparticle ensemble as $\rho(t)=$ $2 N_{+}(t) / N-1$. Let us define also the state of that ensemble. We assume that the state of the nanoparticle ensemble at time $t$ is known if the directions of all magnetic moments are known, i.e., we describe the ensemble state by the set of signs $\sigma_{i}(t) \equiv \sigma_{i}(i=1, \ldots, N)$, where $\sigma_{i}=+$ or - depending on whether the vector $\mathbf{m}_{i}(t)$ fluctuates around the positive or negative direction of the $z$-axis.

Given the ensemble state, neglecting the fluctuations of $\mathbf{m}_{i}(t)$, and taking into account that approximately $\mathbf{m}_{i}(t)=\sigma_{i} m \mathbf{e}_{z}$ for the time intervals between the reorientations, we can write the local dipolar field $\mathbf{h}_{i}(t)$ acting on the magnetic moment $\mathbf{m}_{i}(t)$ as $\mathbf{h}_{i}(t)=h_{i}(t) \mathbf{e}_{z}$. Here

$$
h_{i}(t)=-m \sum_{j \neq i} \sigma_{j} \frac{1}{r_{i j}^{3}},
$$

$m=\left|\mathbf{m}_{i}(t)\right|, \mathbf{e}_{z}$ is the unit vector along the $z$-axis, and $r_{i j}$ is the distance between the centers of corresponding nanoparticles. If at time $t$ the magnetic moments do not undergo reorientations, then each nanoparticle is under the influence of the local dipolar field (2.1). Even if some magnetic moments are reoriented, their number is much less than $N$ because $\varepsilon_{i} \gg 1$, and formula (2.1) remains approximately valid. For sufficiently small times intervals we can consider therefore the ensemble of interacting nanoparticles as a system of independent magnetic moments, each of which feels its own external magnetic field $\mathbf{h}_{i}(t)$. This fact significantly simplifies the numerical investigation of the magnetic relaxation in ensembles of dipolar interacting nanoparticles.

Let us assume that the probabilities of reorientation per unit time $w_{\sigma_{j}}(t ; j)$ (i.e., the probability densities of reorientation) of the vectors $\mathbf{m}_{j}(t)(j=1, \ldots, N)$ from the positive direction of the $z$-axis (if $\sigma_{j}=+$ ) and from the negative one (if $\sigma_{j}=-$ ) are known. We 
also assume that on the interval $(t, t+\tau)$ the probabilities of two and more reorientations of $\mathbf{m}_{j}(t)$ are negligibly small. Then, taking into account that $N_{+}(t+\tau)-N_{+}(t)$ is equal to the difference between the number of reorientations from the negative direction of the $z$-axis and the number of reorientations from the positive direction of the $z$-axis, we obtain

$$
\rho(t+\tau)-\rho(t)=-\frac{2 \tau}{N} \sum_{j=1}^{N} \sigma_{j} w_{\sigma_{j}}(t ; j) .
$$

The probability densities $w_{\sigma_{j}}(t ; j)$ depend on the local field $h_{j}(t)$, and Eq. (2.2) can be applied if the ensemble state at time $t$ is known. However Eq. (2.2) is not an iterative equation for the ensemble state; it only defines $\rho(t+\tau)$ but not the ensemble state at time $t+\tau$. In order to use Eq. (2.2) as the recurrence equation for the calculation of the law of magnetic relaxation, we need to determine the values $w_{\sigma_{j}}(t ; j)$ and develop a procedure to find the state of the nanoparticle ensemble at time $t+\tau$, if its state at time $t$ is known. We will describe that procedure in the next section. Below we calculate the probability densities $w_{\sigma_{j}}(t ; j)$.

\section{B. Probability densities of reorientation}

The probability densities of reorientation are given by $w_{\sigma_{j}}(t ; j)=1 / t_{s}^{\sigma_{j}}(t ; j)$, where $t_{s}^{\sigma_{j}}(t ; j)$ are the mean times that the magnetic moment $\mathbf{m}_{j}(t)$ spends pointing in the positive (when $\sigma_{j}=+$ ) and the negative (when $\sigma_{j}=-$ ) directions of the $z$-axis. These times can be represented as $t_{s}^{\sigma_{j}}(t ; j)=2 t_{m}^{\sigma_{j}}(t ; j)$, where $t_{m}^{\sigma_{j}}(t ; j)$ are the mean times for $\mathbf{m}_{j}(t)$ to reach for the first time the state with a maximum value of the nanoparticle magnetic energy $W_{j}$. The factor 2 takes into account the fact that from that state the magnetic moment $\mathbf{m}_{j}(t)$ can transit to the state $\sigma_{j}=+$ or $\sigma_{j}=-$ with probability $1 / 2$. In our case, the magnetic energy $W_{j}$ includes the anisotropy energy $-\left(H_{a} / 2 m\right) m_{j z}^{2}(t)$ and the Zeeman energy $-h_{j}(t) m_{j z}(t)$, so that it has axial symmetry and

$$
\begin{aligned}
W_{j} & \equiv W_{j}\left(\theta_{j}(t), t\right) \\
& =-\frac{1}{2} H_{a} m\left[\cos ^{2} \theta_{j}(t)+2 b_{j}(t) \cos \theta_{j}(t)\right] .
\end{aligned}
$$

Here $H_{a}$ is the anisotropy field, $\theta_{j}(t)$ is the polar angle of $\mathbf{m}_{j}(t)$, and $b_{j}(t)=h_{j}(t) / H_{a}$ (assuming that two equilibrium directions exist for each magnetic moment, $\left|b_{j}(t)\right|<1$ for all 
nanoparticles). Accordingly, the state corresponding to the maximum value of $W_{j}$ is defined by the polar angle

$$
\Omega_{j}(t)=\arccos \left[-b_{j}(t)\right]
$$

From the mathematical point of view, the calculation of the mean times $t_{m}^{\sigma_{j}}(t ; j)$ is a particular case of a general problem, known in the theory of Markovian processes as the first-passage time problem. ${ }^{28}$ In our case, the Markovian process is the vector $\mathbf{m}_{j}(t)$, and the level set of first passages for $\mathbf{m}_{j}(t)$ is the conical surface defined by Eq. (2.4). We describe the dynamics of the nanoparticle magnetic moments $\mathbf{m}_{j}(t) \equiv \mathbf{m}_{j}$ by the system of stochastic Landau-Lifshitz equations

$$
\dot{\mathbf{m}}_{j}=-\gamma \mathbf{m}_{j} \times\left(\mathbf{H}_{j}+\mathbf{n}_{j}\right)-\frac{\lambda \gamma}{m} \mathbf{m}_{j} \times\left(\mathbf{m}_{j} \times \mathbf{H}_{j}\right),
$$

where $j=1, \ldots, N, \gamma(>0)$ is the gyromagnetic ratio, $\lambda(\ll 1)$ is the damping parameter,

$$
\mathbf{H}_{j} \equiv-\frac{\partial W_{j}(t)}{\partial \mathbf{m}_{j}}=H_{a}\left[\cos \theta_{j}(t)+b_{j}(t)\right] \mathbf{e}_{z}
$$

is the effective magnetic field acting on $\mathbf{m}_{j}$, and $\mathbf{n}_{j}=\mathbf{n}_{j}(t)$ is the thermal magnetic field that models the action of the thermostat. The thermal field is approximated by Gaussian white noise with zero mean values $\overline{\mathbf{n}_{j}(t)}=0$ [the overbar denotes averaging with respect to the sample paths of $\mathbf{n}_{j}(t)$ ] and correlations functions

$$
\overline{n_{i \alpha}\left(t_{1}\right) n_{j \beta}\left(t_{2}\right)}=2 \Delta \delta_{i j} \delta_{\alpha \beta} \delta\left(t_{2}-t_{1}\right)
$$

Here $n_{i \alpha}(t)(\alpha=x, y, z)$ are the Cartesian components of $\mathbf{n}_{i}(t), \Delta=\lambda k_{B} T / \gamma m$ is the intensity of the thermal magnetic field, $\delta_{i j}$ is the Kronecker symbol, and $\delta(t)$ is the Dirac $\delta$ function.

If we treat the local dipolar fields $\mathbf{h}_{j}(t)$ as external magnetic fields, then we can consider the nanoparticles to be independent. In other words, in this case the stochastic LandauLifshitz equations (2.5) are independent, and the dynamics of each magnetic moment is described separately. Let $P_{j}=P_{j}\left(\vartheta_{j}, t \mid \vartheta_{j}^{\prime}, t^{\prime}\right)$ be the conditional probability density that $\theta_{j}(t)=\vartheta_{j}$ given that $\theta_{j}\left(t^{\prime}\right)=\vartheta_{j}^{\prime}\left(t \geq t^{\prime}\right)$. [Note that in the case of axial symmetry $P_{j}$ does not depend on the azimuthal angle of $\mathbf{m}_{j}$.] Then, using the Stratonovich interpretation ${ }^{29}$ of Eq. (2.5) and applying standard methods, ${ }^{28}$ we can write for $P_{j}$ the forward Fokker-Planck equation

$$
\frac{\partial P_{j}}{\partial t}=\frac{\partial}{\partial \vartheta_{j}}\left[\frac{\lambda \gamma}{m} \frac{\partial W_{j}\left(\vartheta_{j}, t\right)}{\partial \vartheta_{j}}-\Delta \gamma^{2} \cot \vartheta_{j}\right] P_{j}
$$




$$
+\Delta \gamma^{2} \frac{\partial^{2} P_{j}}{\partial \vartheta_{j}^{2}}
$$

and the backward Fokker-Planck equation

$$
\begin{aligned}
\frac{\partial P_{j}}{\partial t^{\prime}}= & {\left[\frac{\lambda \gamma}{m} \frac{\partial W_{j}\left(\vartheta_{j}^{\prime}, t^{\prime}\right)}{\partial \vartheta_{j}^{\prime}}-\Delta \gamma^{2} \cot \vartheta_{j}^{\prime}\right] \frac{\partial P_{j}}{\partial \vartheta_{j}^{\prime}} } \\
& -\Delta \gamma^{2} \frac{\partial^{2} P_{j}}{\partial \vartheta_{j}^{\prime 2}} .
\end{aligned}
$$

As a rule, the study of the magnetic properties of nanoparticle ensembles is based on forward Fokker-Planck equations similar to Eq. (2.8), which allow us to express the statistical characteristics of ensembles as functions of time $t$. At the same time, backward Fokker-Planck equations are very useful to describe the thermally induced reversal of the nanoparticle magnetic moments. ${ }^{30}$ We use the backward Fokker-Planck equation (2.9) to calculate the mean first-passage times $t_{m}^{\sigma_{j}}(t ; j)$.

To use Eq. (2.2) as the recurrence equation for finding the reduced magnetization at the discrete times $t=t_{n}\left(n=0,1, \ldots, M, t_{0}=0, t_{n+1}>t_{n}\right)$, we need to calculate $t_{m}^{\sigma_{j}}\left(t_{n} ; j\right)$ for $n=$ $0,1, \ldots, M-1$. Since to each time $t_{n}$ corresponds the angle $\Omega_{j}\left(t_{n}\right)$, it is necessary in Eq. (2.9) to replace $W_{j}\left(\vartheta_{j}^{\prime}, t^{\prime}\right)$ by $W_{j}\left(\vartheta_{j}^{\prime}, t_{n}\right)$. In other words, to find $t_{m}^{\sigma_{j}}\left(t_{n} ; j\right)$ we must use Eq. (2.9) with an energy term $W_{j}\left(\vartheta_{j}^{\prime}, t^{\prime}\right)$ that does not depend on $t^{\prime}$. This important requirement results in a condition of homogeneity for the random process $\theta_{j}(t), P_{j}\left(\vartheta_{j}, t \mid \vartheta_{j}^{\prime}, t^{\prime}\right)=P_{j}\left(\vartheta_{j}, t-\right.$ $\left.t^{\prime} \mid \vartheta_{j}^{\prime}, 0\right)$ and significantly simplifies the problem.

To calculate $t_{m}^{\sigma_{j}}\left(t_{n} ; j\right)$, we first introduce the mean times $T_{j}=T_{j}^{\sigma_{j}}\left(\vartheta_{j}^{\prime} ; t_{n}\right)$, the time necessary for $\theta_{j}(t)\left[\theta_{j}(0)=\vartheta_{j}^{\prime}, \vartheta_{j}^{\prime} \in\left(0, \Omega_{j}\left(t_{n}\right)\right)\right.$ if $\sigma_{j}=+$, and $\vartheta_{j}^{\prime} \in\left(\Omega_{j}\left(t_{n}\right), \pi\right)$ if $\left.\sigma_{j}=-\right]$ to first reach the angle $\Omega_{j}\left(t_{n}\right)$. The desired times are expressed through $T_{j}$ as

$$
t_{m}^{\sigma_{j}}\left(t_{n} ; j\right)=T_{j}^{\sigma_{j}}\left(\pi\left(1-\sigma_{j} 1\right) / 2 ; t_{n}\right)
$$

and the values $T_{j}$ themselves are represented in the form

$$
\begin{aligned}
T_{j}= & \int_{0}^{\infty} d u \int_{\Omega_{j}\left(t_{n}\right)\left(1-\sigma_{j} 1\right) / 2}^{\pi\left(1-\sigma_{j} 1\right) / 2+\Omega_{j}\left(t_{n}\right)\left(1+\sigma_{j} 1\right) / 2} d \vartheta \\
& \times P_{j}\left(\vartheta, u \mid \vartheta_{j}^{\prime}, 0\right) .
\end{aligned}
$$

Taking into account the initial condition $P_{j}\left(\vartheta_{j}, 0 \mid \vartheta_{j}^{\prime}, 0\right)=\delta\left(\vartheta_{j}-\vartheta_{j}^{\prime}\right)$, the homogeneity condition $P_{j}\left(\vartheta_{j}, t \mid \vartheta_{j}^{\prime}, t^{\prime}\right)=P_{j}\left(\vartheta_{j}, t-t^{\prime} \mid \vartheta_{j}^{\prime}, 0\right)$, and the expression (2.3), we obtain after integration of both sides of the modified equation (2.9) over $u=t-t^{\prime}$ and $\vartheta=\vartheta_{j}$ as in Eq. (2.11) the 
ordinary differential equation for $T_{j}$

$$
\frac{d^{2} T_{j}}{d \vartheta_{j}^{\prime 2}}+\left[\cot \vartheta_{j}^{\prime}-2 a\left(b_{j}\left(t_{n}\right)+\cos \vartheta_{j}^{\prime}\right) \sin \vartheta_{j}^{\prime}\right] \frac{d T_{j}}{d \vartheta_{j}^{\prime}}=-a t_{r}
$$

$\left(a=H_{a} m / 2 k_{B} T, t_{r}=2 / \lambda \gamma H_{a}\right)$.

To find the unique solution of Eq. (2.12), we need to impose two boundary conditions for the mean times $T_{j}$. The first condition follows immediately from the definition of these times: $\left.T_{j}\right|_{\vartheta_{j}^{\prime}=\Omega_{j}\left(t_{n}\right)}=0$. We can find the second by analysing the solutions of Eq. (2.12) for small vicinities of the angles $\vartheta_{j}^{\prime}=0$ and $\vartheta_{j}^{\prime}=\pi$. There Eq. (2.12) is reduced to

$$
\frac{d^{2} T_{j}}{d \vartheta_{j}^{\prime 2}}+\frac{1}{\vartheta_{j}^{\prime}-\pi\left(1-\sigma_{j} 1\right) / 2} \frac{d T_{j}}{d \vartheta_{j}^{\prime}}=-a t_{r},
$$

and its general solution is given by

$$
\begin{aligned}
T_{j}= & c_{j} \ln \left|\vartheta_{j}^{\prime}-\pi\left(1-\sigma_{j} 1\right) / 2\right|+d_{j} \\
& -a t_{r}\left[\vartheta_{j}^{\prime}-\pi\left(1-\sigma_{j} 1\right) / 2\right]^{2} / 4
\end{aligned}
$$

where $c_{j}$ and $d_{j}$ are constants of integration. Since $T_{j}$ are bounded quantities, the condition $c_{j}=0$ must hold. This condition can be represented equivalently in the form of the second boundary condition: $d T_{j} /\left.d \vartheta_{j}^{\prime}\right|_{\vartheta_{j}^{\prime}=\pi\left(1-\sigma_{j} 1\right) / 2}=0$. Note that these boundary conditions correspond to the case where so-called absorbing and reflecting barriers ${ }^{28}$ are placed at the points $\vartheta_{j}^{\prime}=\Omega_{j}\left(t_{n}\right)$ and $\vartheta_{j}^{\prime}=0, \pi$, respectively.

Solving Eq. (2.12) with these boundary conditions by the method of variation of constants $^{31}$ and using the representation (2.10), we obtain the rigorous formula

$$
\begin{aligned}
t_{m}^{\sigma_{j}}\left(t_{n} ; j\right)= & a t_{r} \int_{-\sigma_{j} b_{j}\left(t_{n}\right)}^{1} d x \frac{e^{-a\left[x+\sigma_{j} b_{j}\left(t_{n}\right)\right]^{2}}}{1-x^{2}} \\
& \times \int_{x}^{1} d y e^{a\left[y+\sigma_{j} b_{j}\left(t_{n}\right)\right]^{2}}
\end{aligned}
$$

which is valid for arbitrary $a$ and $\left|b_{j}\left(t_{n}\right)\right|<1$. Using Eq. (2.15), let us calculate $w_{\sigma_{j}}\left(t_{n} ; j\right)$ for $\varepsilon_{j} \gg 1$. According to Eq. (2.3), the heights $\Delta U_{j}^{\sigma_{j}}(t)$ of the potential barrier between the equilibrium directions of $\mathbf{m}_{j}$ can be written in the form $\Delta U_{j}^{\sigma_{j}}(t)=\frac{1}{2} H_{a} m\left[1+\sigma_{j} b_{j}(t)\right]^{2}$, and, since $\Delta U_{j}=\min \Delta U_{j}^{\sigma_{j}}(t)$, the condition $\varepsilon_{j} \gg 1$ leads to $a\left[1+\sigma_{j} b_{j}\left(t_{n}\right)\right]^{2} \gg 1$. Taking into account that the asymptotic formulas

$$
\begin{aligned}
\int_{x}^{1} d y e^{a\left[y+\sigma_{j} b_{j}\left(t_{n}\right)\right]^{2}} & =\frac{e^{a\left[1+\sigma_{j} b_{j}\left(t_{n}\right)\right]^{2}}}{2 a\left[1+\sigma_{j} b_{j}\left(t_{n}\right)\right]}, \\
\int_{-\sigma_{j} b_{j}\left(t_{n}\right)}^{1} d x \frac{e^{-a\left[x+\sigma_{j} b_{j}\left(t_{n}\right)\right]^{2}}}{1-x^{2}} & =\frac{1}{2} \sqrt{\frac{\pi}{a}} \frac{1}{1-b_{j}^{2}\left(t_{n}\right)}
\end{aligned}
$$


hold as $a\left[1+\sigma_{j} b_{j}\left(t_{n}\right)\right]^{2} \rightarrow \infty$, we find in the same limit

$$
\begin{aligned}
w_{\sigma_{j}}\left(t_{n} ; j\right)= & \frac{2}{t_{r}} \sqrt{\frac{a}{\pi}}\left[1-b_{j}^{2}\left(t_{n}\right)\right]\left[1+\sigma_{j} b_{j}\left(t_{n}\right)\right] \\
& \times e^{-a\left[1+\sigma_{j} b_{j}\left(t_{n}\right)\right]^{2}} .
\end{aligned}
$$

Note that Eq. (2.16) follows also from Brown's results ${ }^{3}$ for isolated nanoparticles in a longitudinal external field obtained with the forward Fokker-Planck equation for $\varepsilon_{j} \gg 1$. We have presented here an alternative derivation of Eq. (2.16) based on the backward FokkerPlanck equation, because within this approach the mean first-passage times $t_{m}^{\sigma_{j}}(t ; j)$ and the probability densities of reorientation $w_{\sigma_{j}}\left(t_{n} ; j\right)$ are calculated exactly for arbitrary $\varepsilon_{j}$.

If the condition $\varepsilon_{j} \gg 1$ holds for all nanoparticles and the ensemble state at time $t=t_{n}$ is known, then for the same time we can find the dipolar fields acting on each nanoparticle, using the formula (2.1), and calculate the probability densities of reorientation of each magnetic moment, using the formula (2.16).

\section{Mean-field approximation}

To illustrate the influence of the correlations of the magnetic moments and of the finite size of the nanoparticle ensemble on the magnetic relaxation, we must first calculate the relaxation law $\rho_{m f}(t)$ for an infinite lattice within the mean-field approximation. To this end, we derive the equation that this relaxation law satisfies, based on the results obtained above. Since within the mean-field approximation the same mean dipolar field acts on all magnetic moments, it is necessary in Eq. (2.16) to replace $b_{j}(t)$ (we drop the index $n$ in $t_{n}$ ) by $b(t)=\overline{b_{j}(t)}$. This implies that all magnetic moments for which $\sigma_{j}=+$ and all magnetic moments for which $\sigma_{j}=-$ are reoriented with the same probability densities, $w_{+}(t)$ and $w_{-}(t)$ respectively, where

$$
w_{ \pm}(t)=\frac{2}{t_{r}} \sqrt{\frac{a}{\pi}}\left[1-b^{2}(t)\right][1 \pm b(t)] e^{-a[1 \pm b(t)]^{2}} .
$$

The function $b(t)$ is given by ${ }^{20}$

$$
b(t)=-9.034 \frac{m}{H_{a} d^{3}} \rho_{m f}(t)
$$

therefore the probability densities $w_{ \pm}(t)$ depend on $t$ only via the reduced magnetization $\rho_{m f}(t)$, i.e., $w_{ \pm}(t)=w_{ \pm}\left(\rho_{m f}(t)\right)$. Finally, using the equality $\sum_{j} \sigma_{j}=N_{+}(t)-N_{-}(t)$ and the 
definition of $\rho(t)$, we obtain from Eq. (2.2) for $\tau \rightarrow 0$ and $N \rightarrow \infty$ the required differential equation

$$
\dot{\rho}_{m f}(t)=-\rho_{m f}(t)\left[w_{+}(t)+w_{-}(t)\right]-w_{+}(t)+w_{-}(t)
$$

$\left(\rho_{m f}(0)=1\right)$, which defines the law of magnetic relaxation in the mean-field approximation. Note that the same equation follows from the solution of the forward Fokker-Planck equation $(2.8) \cdot{ }^{20}$

Calling the right hand side of Eq. (2.19) $-F\left(\rho_{m f}(t)\right)$, we can reduce this equation to the integral form

$$
\int_{\rho_{m f}(t)}^{1} \frac{d x}{F(x)}=t
$$

Its solution for small and large times yields ${ }^{20} \rho_{m f}(t)=1-t / \tau_{0}$ and $\rho_{m f}(t) \propto \exp \left(-t / \tau_{\infty}\right)$, respectively, where

$$
\tau_{0}=t_{r} \sqrt{\frac{\pi}{a}} \frac{e^{a(1-\xi)^{2}}}{4\left(1-\xi^{2}\right)(1-\xi)}
$$

is the initial relaxation time,

$$
\tau_{\infty}=t_{r} \sqrt{\frac{\pi}{a}} \frac{e^{a}}{4[1+(2 a-1) \xi]}
$$

is the final relaxation time, and $\xi=-b(0)(0 \leq \xi<1)$ is a parameter characterizing the intensity of dipolar interaction on an infinite lattice. According to Eqs. (2.21) and (2.22), the relaxation process in ensembles of dipolar interacting nanoparticles is approximately characterized by two relaxation times, $\tau_{0}$ and $\tau_{\infty}$, while in the case of non-interacting nanoparticles, i.e., $\xi=0$, it is characterized by the single relaxation time $\tau_{n}=t_{r} \sqrt{\pi / 16 a} \exp a$. Since $\tau_{n}>\tau_{0}$ and $\tau_{n}>\tau_{\infty}$, the dipolar interaction enhances relaxation, and since $\tau_{0}<\tau_{\infty}$, the relaxation rate decreases with time. For ensembles where the value of $\xi$ is not too small, the strong inequality $\tau_{0} \ll \tau_{\infty}$ usually holds, and the decrease can be very large.

Note that the description of magnetic relaxation based on Eqs. (2.2) and (2.19) is valid if the quasiequilibrium distribution of the nanoparticle magnetic moments is established, i.e., if $^{20} t \gtrsim t_{q e} \sim a t_{r}$. In other words, these equations describe the slow phase of magnetic relaxation. For $t \sim t_{q e}$, the probability of reorientation of the nanoparticle magnetic moments from the initial state is vanishingly small. Therefore we can transfer the origin of time to an arbitrary point $t \sim t_{q e}$ and, since for $a>>1$ and $t \sim t_{q e}$ the approximate equalities $\overline{m_{j z}(t)} \approx m$ hold, use the initial conditions $\rho(0)=1$ and $\rho_{m f}(0)=1$. 


\section{NUMERICAL SIMULATIONS}

\section{A. The computational algorithm}

According to the results of the previous section, to compute the law of magnetic relaxation in some time interval $\left(0, t_{M}\right)$ it is necessary to know the states of the nanoparticle ensemble at the discrete times $t=t_{n}(n=0,1, \ldots, M-1)$. The state for $n=0$, i.e., for $t=0$, is known from the initial condition: $\sigma_{j}(0)=+$ for all $j$. To find the state at any other time we proceed as follows. First we assume that the state of the nanoparticle ensemble at the time $t=t_{n}$ is known. This means that the set $A_{+}\left(t_{n}\right)$ of numbers $j$ for which $\sigma_{j}\left(t_{n}\right)=+$, and the set $A_{-}\left(t_{n}\right)$ of numbers $j$ for which $\sigma_{j}\left(t_{n}\right)=-$ are fully defined. It is evident that the set $A_{+}\left(t_{n}\right)$ contains $N_{+}\left(t_{n}\right)$ elements, and the set $A_{-}\left(t_{n}\right)$ contains $N_{-}\left(t_{n}\right)$ elements.

Next, assuming that the time interval $\Delta t_{n+1}=t_{n+1}-t_{n}$ is small enough, we introduce the average numbers of reorientations

$$
\nu_{ \pm}\left(t_{n}, t_{n+1}\right)=\Delta t_{n+1} \sum_{j \in A_{ \pm}\left(t_{n}\right)} w_{ \pm}\left(t_{n} ; j\right)
$$

that occur during $\Delta t_{n+1}$ for the sets of positively (upper sign) and negatively (lower sign) oriented magnetic moments. Strictly speaking, Eq. (3.1) is valid if the strong inequality $\Delta t_{n+1} \max \left\{w_{ \pm}\left(t_{n} ; j\right)\right\} \ll 1$ holds. Its use can drastically increase the time required for the computation of the relaxation law in some cases. Therefore, instead of the exact representation (3.1) we use the approximate one

$$
\nu_{ \pm}\left(t_{n}, t_{n+1}\right)=\sum_{j \in A_{ \pm}\left(t_{n}\right)} U\left(\Delta t_{n+1} w_{ \pm}\left(t_{n} ; j\right)\right)
$$

$[U(x)=x$ if $x \leq 1$, and $U(x)=1$ if $x>1]$, which is valid if the weaker condition $\nu_{ \pm}\left(t_{n}, t_{n+1}\right) \ll N$ holds, and from Eq. (2.2) we obtain

$$
\rho\left(t_{n+1}\right)=\rho\left(t_{n}\right)-\frac{2}{N}\left[\nu_{+}\left(t_{n}, t_{n+1}\right)-\nu_{-}\left(t_{n}, t_{n+1}\right)\right] .
$$

Equations (3.3), (3.2), (2.16), and (2.1) allow us to calculate the reduced magnetization at time $t=t_{n+1}$, if the nanoparticle state at time $t=t_{n}$ is known. To find the nanoparticle state at time $t=t_{n+1}$, we need to choose the sites where the magnetic moments must be reoriented. To reflect the random character of the thermal fluctuations, these sites should be chosen randomly, while at the same time preference should be given to those sites that have 
larger probabilities of reorientation. To satisfy both requirements we proceed in the following way. First we choose the time steps $\Delta t_{n+1}$. Since the number of magnetic moments that are reoriented per unit time can appreciably decrease with time, we select steps of varying length, $\Delta t_{n+1}=\eta\left[w_{+}\left(t_{n}\right)+w_{-}\left(t_{n}\right)\right]^{-1}$. The parameter $\eta$ must be chosen small enough to satisfy the condition $\nu_{ \pm}\left(t_{n}, t_{n+1}\right) \ll N$ (in our calculations $\left.\eta=5 \times 10^{-3}\right)$. Then we calculate the values $\Delta t_{n+1} w_{+}\left(t_{n} ; j\right)$ for $j \in A_{+}\left(t_{n}\right)$, and using the formula (3.2) we find the average number of reorientations

$$
\nu_{+}\left(t_{n}, t_{n+1}\right)=r_{+}\left(t_{n}, t_{n+1}\right)+\Delta t_{n+1} \sum_{j \in A_{+}^{\prime}\left(t_{n}\right)} w_{+}\left(t_{n} ; j\right)
$$

that occur during the time interval $\Delta t_{n+1}$ in the set of positively oriented magnetic moments. Here $r_{+}\left(t_{n}, t_{n+1}\right)$ is the number of lattice sites where $\Delta t_{n+1} w_{+}\left(t_{n} ; j\right)>1$, and $A_{+}^{\prime}\left(t_{n}\right)$ is the set of lattice sites where $\Delta t_{n+1} w_{+}\left(t_{n} ; j\right) \leq 1$. Further, we introduce the number of reorientations as $n_{+}\left(t_{n}, t_{n+1}\right)=\left[\nu_{+}\left(t_{n}, t_{n+1}\right)\right]+I$, where $\left[\nu_{+}\left(t_{n}, t_{n+1}\right)\right]$ is the integer part of $\nu_{+}\left(t_{n}, t_{n+1}\right)$, and $I=0$ or 1 with probability $p_{0}=\nu_{+}\left(t_{n}, t_{n+1}\right)-\left[\nu_{+}\left(t_{n}, t_{n+1}\right)\right]$ and $p_{1}=1-p_{0}$, respectively. Using a random number generator, we obtain a value for $n_{+}\left(t_{n}, t_{n+1}\right)$.

Among the $n_{+}\left(t_{n}, t_{n+1}\right)$ magnetic moments that must be inverted at time $t=t_{n+1}$, we immediately invert the $r_{+}\left(t_{n}, t_{n+1}\right)$ magnetic moments at lattice sites where the condition $\Delta t_{n+1} w_{+}\left(t_{n} ; j\right)>1$ holds. [Recall that a one-to-one correspondence exists between the lattice sites and numbers $j$.] To find the remaining $n_{+}\left(t_{n}, t_{n+1}\right)-r_{+}\left(t_{n}, t_{n+1}\right)$ lattice sites where the magnetic moments have to be inverted, we first generate a random number that lies in the interval of length $\sum_{j \in A_{+}^{\prime}\left(t_{n}\right)} w_{+}\left(t_{n} ; j\right)$. This interval contains $N_{+}\left(t_{n}\right)-r_{+}\left(t_{n}, t_{n+1}\right)$ subintervals of lengths $w_{+}\left(t_{n} ; j\right)$. We store the number $j$ of the subinterval (i.e., the position of the site) that contains the random number in memory, and then that subinterval is removed. Next we generate a random number that lies in the new interval formed by the remaining subintervals. The number $j$ of the subinterval that contains this random number is again stored in memory, and then this subinterval is also removed. Iterating this procedure $n_{+}\left(t_{n}, t_{n+1}\right)-r_{+}\left(t_{n}, t_{n+1}\right)$ times, we find all $n_{+}\left(t_{n}, t_{n+1}\right)$ lattice sites where positively oriented magnetic moments must be inverted at time $t=t_{n+1}$.

Introducing in the same way the average number of reorientations $\nu_{-}\left(t_{n}, t_{n+1}\right)$ that occur in the set of negatively oriented magnetic moments, and using the procedure described above, we determine $n_{-}\left(t_{n}, t_{n+1}\right)$ lattice sites where these magnetic moments must be inverted at time $t=t_{n+1}$. Since the ensemble state at time $t=t_{n}$ is known, the ensemble state at 
$t=t_{n+1}$, i.e., after the inversion of $n_{+}\left(t_{n}, t_{n+1}\right)+n_{-}\left(t_{n}, t_{n+1}\right)$ magnetic moments on well defined lattice sites, is known too. Taking the latter state as the initial state, we can find in the same manner the ensemble state at time $t=t_{n+2}$, and so on.

Using the known state of the nanoparticle ensemble at the initial time $t=0$ and applying the algorithm described above, we can find the states for all times $t=t_{n}(n=1, \ldots, M-1)$. Since our algorithm is a probabilistic one, the reduced magnetization calculated by the formula (3.3) is a random quantity. Let us designate that random reduced magnetization in the $k$ th numerical experiment as $\rho_{s i m}^{k}\left(t_{n}\right)$. [A numerical experiment consists of one application of the algorithm to determine the ensemble states at all times $t=t_{n}$. Then we define the numerically simulated relaxation law as

$$
\rho_{\text {sim }}\left(t_{n}\right)=\frac{1}{K} \sum_{k=1}^{K} \rho_{\text {sim }}^{k}\left(t_{n}\right),
$$

where $K$ is the number of numerical experiments. To avoid any misunderstanding, we emphasize that within the proposed algorithm the dipolar field (2.1) is calculated exactly, and it is re-calculated after each time step.

\section{B. Numerical results and discussion}

We have used our analytical results and the numerical algorithm described above to study the role that the finite size of the nanoparticle ensemble and the correlations of the nanoparticle magnetic moments play in magnetic relaxation. We found that the reduced magnetization $\rho_{\text {sim }}(t)(t>0)$ decreases, when the parameter $L$, a measure of the ensemble size, increases, i.e., $\left.\rho_{\text {sim }}(t)\right|_{L_{1}}>\left.\rho_{\text {sim }}(t)\right|_{L_{2}}$ if $L_{2}>L_{1}$, and $\left.\rho_{\text {sim }}(t)\right|_{L}$ tends to the limiting value $\rho_{\text {lim }}(t)$ as $L \rightarrow \infty$. We explain such behavior of $\rho_{\text {sim }}(t)$ as follows. Increasing $L$ leads to an increase, on average, of the local dipolar fields acting on the nanoparticle magnetic moments. As a result, the average of the probability densities of reorientation of the positively oriented magnetic moments increases, and the average of the probability densities of reorientation of the negatively oriented magnetic moments decreases. According to Eq. (2.2), this means that $\rho_{\text {sim }}(t)$ decreases when $L$ grows.

To verify this statement, we have calculated $\rho_{\text {sim }}(t)$ for different ensembles of Co nanoparticles characterized by the parameters $H_{a}=6400$ Oe, $m / V=1400 \mathrm{G}$ ( $V$ is the nanoparticle volume), $\lambda=0.2$, and $r=4 \mathrm{~nm}$. As an illustration, the function $\rho_{\text {sim }}(t)$, obtained at 
$T=300 \mathrm{~K}, d=3 r, L=50$, and $K=100$, and the approximate function $\rho_{\text {lim }}(t)$ are shown in Fig. 2. We found the latter function in the same way as $\rho_{\text {sim }}(t)$, but, to exclude boundary effects, we assume that the basic nanoparticle ensemble (for which we chose $L=100$ ) is surrounded by eight identical ensembles, and each nanoparticle from the basic ensemble is considered as a central one in the square box of the same size (i.e., $L=100$ ) and interacts only with the nanoparticles which belong to this box. In Fig. 2, we also show the function $\rho_{m f}(t)$ calculated via the numerical solution of Eq. (2.19) for an infinite ensemble of Co nanoparticles with the same parameters. Note that in this case $a \approx 29.01, \xi \approx 0.31$, $t_{r} \approx 8.85 \times 10^{-11} \mathrm{~s}, \tau_{0} \approx 1.33 \times 10^{-5} \mathrm{~s}, \tau_{\infty} \approx 1.56 \mathrm{~s}$, and $\tau_{n} \approx 28.89 \mathrm{~s}$.

Since at $t=0$ the local dipolar field for an infinite ensemble is always larger than the highest local dipolar field for a finite one, the condition $\rho_{\text {sim }}(t)>\rho_{m f}(t)(t>0)$ must hold for small enough times. We expect that the same condition holds also for large enough times, since correlations of the nanoparticle magnetic moments lead to slower magnetic relaxation in the final phase than the mean-field theory predicts. As to the relation between $\rho_{\text {sim }}(t)$ and $\rho_{m f}(t)$ at the intermediate times, its character at a fixed temperature depends on the ensemble size, i.e., on the parameter $L$.

To explain this dependence, we note first that at small times magnetic relaxation for finite nanoparticle ensembles occurs faster than in the case where the local dipolar fields are replaced by their average value, i.e., the mean-field approximation. Indeed, in the initial phase of magnetic relaxation only a small number of the nanoparticle magnetic moments is subjected to reorientation. In this case, the reoriented and most of the non-reoriented magnetic moments are under the action of the local dipolar fields, which exceed the mean dipolar field. This means that $w_{-}(t ; j)<w_{-}(t)$ for $j \in A_{-}(t), w_{+}(t ; j)>w_{+}(t)$ for most $j \in A_{+}(t)$, and therefore the actual magnetic relaxation occurs faster than the mean-field approximation predicts. [We emphasize that this conclusion is valid for the initial phase of magnetic relaxation for finite as well as infinite nanoparticle ensembles.] Furthermore, taking into account that an increase in the size of the nanoparticle ensemble leads to an increase, on average, of the local dipolar fields, we expect the following behavior for the dependence of $\rho_{\text {sim }}(t)$ on $L$ (for an illustration, see Fig. 2). If in the nanoparticle ensemble the highest local dipolar field at $t=0$ is small enough in comparison to the case of an infinite ensemble, i.e., if the parameter $L$ does not exceed the critical value $L_{c r}=L_{c r}(T)$, then $\rho_{\text {sim }}(t)>\rho_{m f}(t)$ for all $t>0$ (curve 1 in Fig. 2). At $L=L_{c r}$ the curves $\rho_{\text {sim }}(t)$ and 
$\rho_{m f}(t)$ have a tangency point, and for $L>L_{c r}$ they intersect at times $t=t_{1 \text { in }}$ and $t=t_{2 \text { in }}$ (curve $4, t_{1 \text { in }} \approx 2.25 \times 10^{-5} \mathrm{~s}, t_{2 \text { in }} \approx 2.09 \times 10^{-2} \mathrm{~s}$ ). As $L$ is increased, the time $t_{1 \text { in }}$ of the first intersection decreases, and the time $t_{2 i n}$ of the second one increases. As a result, for $L \rightarrow \infty$ we have $\rho_{\text {sim }}(t) \rightarrow \rho_{\text {lim }}(t), t_{1 \text { in }} \rightarrow 0$, and $t_{2 i n}$ tends to the limiting value $t_{\text {in }}$ (curve $\left.2, t_{\text {in }} \approx 0.46 \mathrm{~s}\right)$.

To characterize the difference between $\rho_{\text {sim }}(t)$ and $\rho_{m f}(t)$, we introduce the parameter $\chi_{L}(t)=\left[\rho_{\text {sim }}(t)-\rho_{m f}(t)\right] / \rho_{\text {sim }}(t)$. Its dependence on $t$ for the same ensembles of Co nanoparticles is shown in Fig. 3. The nonzero value of $\chi_{L}(t)$ is caused by both the finite size of the nanoparticle ensemble and the correlations of the nanoparticle magnetic moments. Correlations significantly change the relaxation law, and their role grows with time, i.e., $\chi_{L}(t) \rightarrow 1$ as $t \rightarrow \infty$.

The fact that the probability densities of reorientation $w_{\sigma_{j}}(t ; j)$, Eq. (2.16) , depend exponentially on the large parameter $a$ has two consequences. The first is obvious, namely, the relaxation law $\rho_{\text {sim }}(t)$ strongly depends on temperature due to the inverse proportionality of $a$ on $T$. The second is more complicated and refers to the time dependence of $\rho_{\text {sim }}(t)$ and $\rho_{m f}(t)$ for different $T$. According to the previous results, if at a certain temperature the parameter $L$ satisfies the condition $L<L_{c r}$, then $\rho_{\text {sim }}(t)>\rho_{m f}(t)$ for all $t>0$. As $T$ decreases, the probability densities $w_{\sigma_{j}}(t ; j)$ decrease with different rates, and the smaller the temperature becomes, the more their relative values differ. This means that as $T$ is reduced, the reorientation of the nanoparticle magnetic moments predominantly occurs at sites where $w_{\sigma_{j}}(t ; j)$ are the largest. As a consequence, for small times the difference between $\rho_{\text {sim }}(t)$ and the relaxation law derived by the mean-field approximation grows as $T$ decreases. Therefore, if at a given temperature the condition $L<L_{c r}$ holds and the values of $L$ and $L_{c r}$ do not differ too much, then the curves $\rho_{s i m}(t)$ and $\rho_{m f}(t)$ can intersect at smaller temperatures. The plots of $\rho_{\text {sim }}(t)$ calculated for ensembles of Co nanoparticles for $L=50$ and $T=300 \mathrm{~K}$ (see Fig. 2), and for $L=50$ and $T=150 \mathrm{~K}$ (see Fig. 4) demonstrate this statement. In the latter case calculations yield $a \approx 58.02, \tau_{0} \approx 10.72 \mathrm{~s}, \tau_{\infty} \approx 2.24 \times 10^{12}$ $\mathrm{s}, \tau_{n} \approx 8.11 \times 10^{13} \mathrm{~s}, t_{1 i n} \approx 56.12 \mathrm{~s}$, and $t_{2 i n} \approx 6.83 \times 10^{11} \mathrm{~s}$.

The relaxation laws calculated above can not be determined using the Monte Carlo method with time step quantification. According to Ref. 27, the time interval $\Delta t$ that 
corresponds to one Monte Carlo step is written in our notations as

$$
\Delta t=\frac{R^{2}\left(1+\lambda^{2}\right) m}{20 k_{B} T \lambda \gamma}
$$

$(R<1)$, and the number $M=\mu \tau_{n} / \Delta t$ of the Monte Carlo steps that are necessary to calculate the relaxation law on the time interval $\left(0, \mu \tau_{n}\right)$ is given by

$$
M=\frac{5 \mu}{R^{2}\left(1+\lambda^{2}\right)} \sqrt{\frac{\pi}{a^{3}}} e^{a} .
$$

For the nanoparticle ensembles considered here, Eq. (3.7) for $R=1$ and $\mu=0.2$ yields $M \approx 4.33 \times 10^{10}$ for $T=300 \mathrm{~K}$, and $M \approx 6.08 \times 10^{22}$ for $T=150 \mathrm{~K}$. Such values of $M$ render of course the use of that method impractical. For comparison, in our approach the number $M$ of time steps $\Delta t_{n+1}$, defined by the condition $\sum_{m=1}^{M} \Delta t_{m}=\mu \tau_{n}$, equals 157 and 169, respectively.

\section{CONCLUSIONS}

We have developed a new method for the numerical simulation of thermally activated magnetic relaxation in $2 \mathrm{D}$ ensembles of uniaxial ferromagnetic nanoparticles whose easy axes of magnetization are perpendicular to their distribution plane. It is based on the analytical determination of the probability densities of reorientation of the nanoparticle magnetic moments and on the numerical determination of the nanoparticle ensemble states for a discrete sequence of times. Using the backward Fokker-Planck equation, we have formulated a rigorous approach to calculate those probability densities, and in the case of high potential barriers between the equilibrium directions of the nanoparticle magnetic moments we have studied the law of magnetic relaxation by this method.

We have shown that magnetic relaxation in finite nanoparticle ensembles can differ strongly from that predicted by the mean-field approximation for infinite ensembles. This difference is caused by the finiteness of the ensemble size as well as correlations between the magnetic moments, which result from the dipolar interaction between nanoparticles. In a finite ensemble, magnetic relaxation for small and large times occurs slower than the mean-field theory predicts for infinite ensembles, and for intermediate times the corresponding relaxation curves, depending on the ensemble size and temperature, can intersect twice. Increase of the ensemble size enhances relaxation, and in the limiting case of an infinite 
ensemble, magnetic relaxation for small times occurs faster and for large times slower than for the mean-field theory. This feature of the relaxation law is caused by the correlation effects whose role grows with time.

\section{ACKNOWLEDGMENTS}

We are grateful to Werner Horsthemke for the critical reading of this manuscript and his valuable comments. This work was supported in part by NATO Grant No. PST.CLG.978108.

* Electronic address: denisov@ssu.sumy.ua

$\dagger$ Electronic address: trohidou@ims.demokritos.gr

1 J. L. Dormann, D. Fiorani, and E. Tronc, Adv. Chem. Phys. 98, 283 (1997).

2 Nanophase Materials: Synthesis-Properties-Applications, edited by G. C. Hadjipanayis and R. W. Siegel (Kluwer, Dordrecht, 1994).

3 W. F. Brown, Jr., Phys. Rev. 130, 1677 (1963).

4 I. Klik and L. Gunther, J. Stat. Phys. 60, 473 (1990).

5 H. B. Braun, Phys. Rev. Lett. 71, 3557 (1993).

6 D. A. Garanin, Phys. Rev. E 54, 3250 (1996).

7 W. T. Coffey, D. S. F. Crothers, J. L. Dormann, Yu. P. Kalmykov, E. C. Kennedy, and W. Wernsdorfer, Phys. Rev. Lett. 80, 5655 (1998).

8 Yu. P. Kalmykov, Phys. Rev. B 61, 6205 (2000).

9 S. Shtrikman and E. P. Wohlfarth, Phys. Lett. A 85, 467 (1981).

10 J. L. Dormann, L. Bessais, and D. Fiorani, J. Phys. C 21, 2015 (1988).

11 S. Mørup and E. Tronc, Phys. Rev. Lett. 72, 3278 (1994).

12 M. F. Hansen and S. Mørup, J. Magn. Magn. Mater. 184, 262 (1998).

13 J. L. Dormann, D. Fiorani, and E. Tronc, J. Magn. Magn. Mater. 202, 251 (1999).

14 R. Prozorov, Y. Yeshurun, T. Prozorov, and A. Gedanken, Phys. Rev. B 59, 6956 (1999).

15 S. I. Denisov, Phys. Solid State 41, 1672 (1999).

16 M. El-Hilo, M. Shatnawy, A. Al-Rsheed, J. Magn. Magn. Mater. 221, 137 (2000). 
17 P. Zhang, F. Zuo, F. K. Urban III, A. Khabari, P. Griffiths, A. Hosseini-Tehrani, J. Magn. Magn. Mater. 225, 337 (2001).

18 The Physics of Ultra-High-Density Magnetic Recording, edited by M. L. Plumer, J. Van Ek, and D. Weller (Springer-Verlag, Berlin, 2001).

19 D. K. Lottis, R. M. White, and E. D. Dahlberg, Phys. Rev. Lett. 67, 362 (1991).

20 S. I. Denisov and K. N. Trohidou, Phys. Rev. B 64, 184433 (2001).

21 S. I. Denisov and K. N. Trohidou, Phys. Status Solidi A 189, 265 (2002).

22 A. Lyberatos and R. W. Chantrell, J. Appl. Phys. 73, 6501 (1993).

23 J. L. García-Palacios and F. J. Lázaro, Phys. Rev. B 58, 14937 (1998).

24 D. V. Berkov, N. L. Gorn, and P. Görnert, Phys. Status Solidi A 189, 409 (2002).

25 J. García-Otero, M. Porto, J. Rivas, and A. Bunde, Phys. Rev. Lett. 84, 167 (2000).

26 L. C. Sampaio, R. Hyndman, F. S. de Menezes, J. P. Jamet, P. Meyer, J. Gierak, C. Chappert, V. Mathet, and J. Ferré, Phys. Rev. B 64, 184440 (2001).

27 U. Nowak, R. W. Chantrell, and E. C. Kennedy, Phys. Rev. Lett. 84, 163 (2000).

28 C. W. Gardiner, Handbook of Stochastic Methods, 2nd ed. (Springer-Verlag, Berlin, 1990).

29 R. L. Stratonovich, SIAM J. Control 4, 362 (1966).

30 S. I. Denisov and A. N. Yunda, Physica B 245, 282 (1998).

31 V. I. Arnol'd, Ordinary Differential Equations, (Springer-Verlag, Berlin, 1992). 


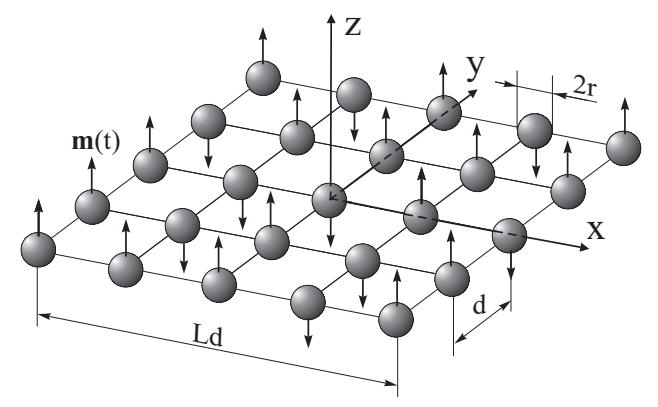

FIG. 1: Schematic representation of the 2D nanoparticle ensemble.

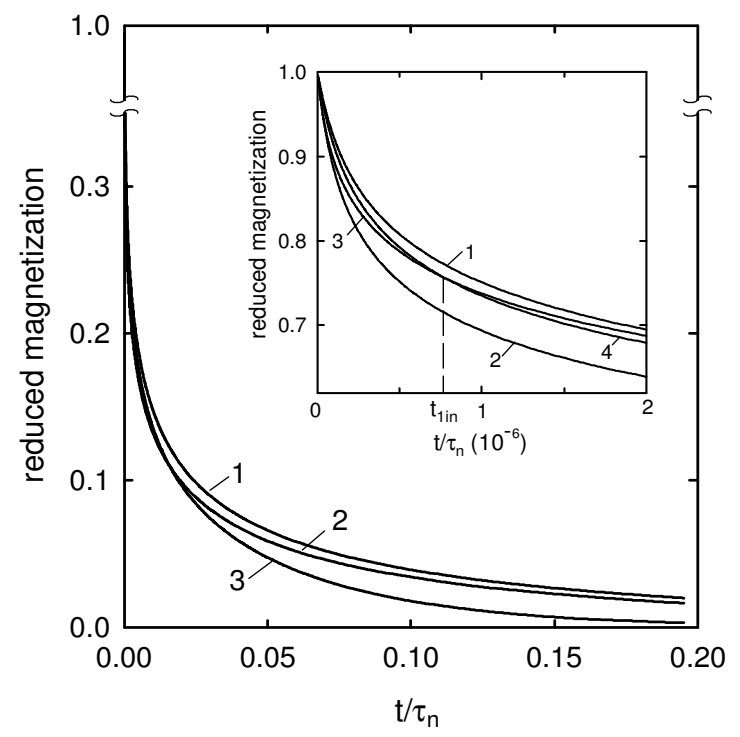

FIG. 2: Plots of $\rho_{\text {sim }}(t)$ for $L=50$ (curve 1), $\rho_{\text {lim }}(t)$ (curve 2), and $\rho_{m f}(t)$ (curve 3). Inset: The same plots and the plot of $\rho_{\text {sim }}(t)$ for $L=70$ (curve 4) for small times. 


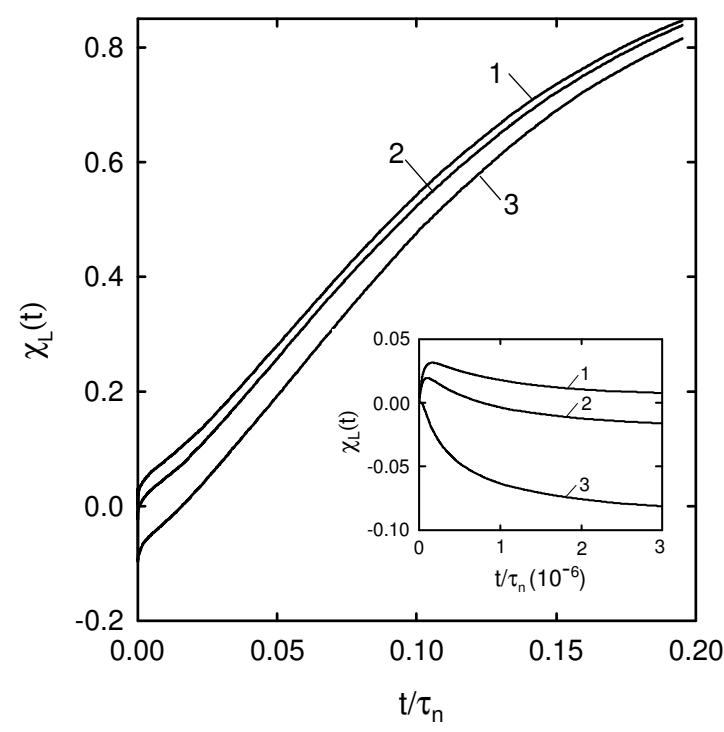

FIG. 3: Plots of $\chi_{L}(t)$ for $L=50$ (curve 1), $L=70$ (curve 2), and $L=\infty$ (curve 3). Inset: The same plots for small times.

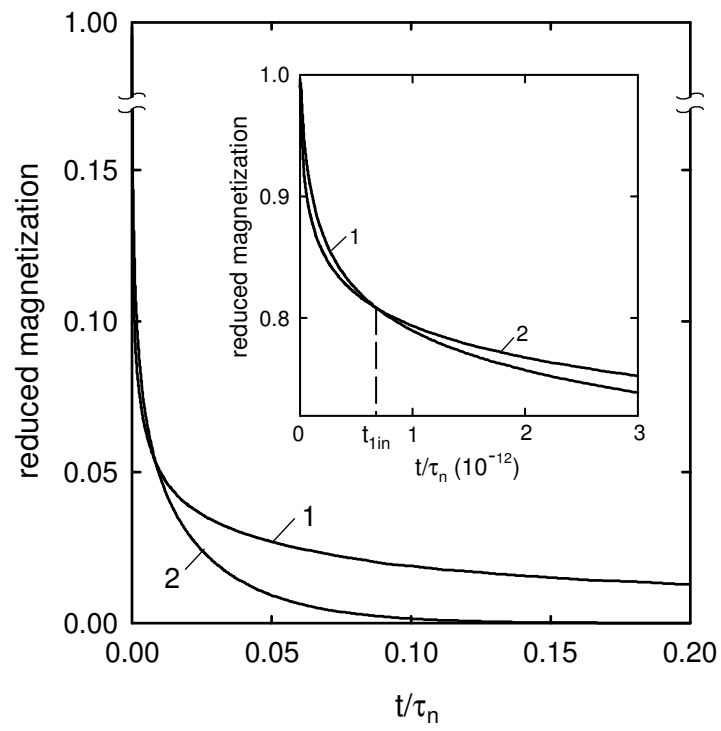

FIG. 4: Plots of $\rho_{\text {sim }}(t)$ (curve 1) and $\rho_{m f}(t)$ (curve 2) for $L=50$ and $T=150 \mathrm{~K}$. Inset: The same plots for small times. 